Original Contribution

\title{
Methadone versus morphine for postoperative pain in patients undergoing surgery for gynecological cancer: A randomized controlled clinical trial ${ }^{\text {th }}$
}

\author{
Sebastiano Mercadante (MD) ${ }^{\mathrm{a}, *}$, Fabrizio David (MD) ${ }^{\mathrm{a}}$, Patrizia Villari (MD) ${ }^{\mathrm{a}}$, \\ Vincenza Manuela Spedale (MD) ${ }^{\mathrm{a}}$, Alessandra Casuccio (BS) ${ }^{\mathrm{b}}$ \\ ${ }^{a}$ Anesthesia and Intensive Care Unit, Pain Relief and Palliative Care Unit, La Maddalena Cancer Center, Palermo, Italy \\ ${ }^{\mathrm{b}}$ Department of Sciences for Health Promotion and Mother Child Care, University of Palermo, Italy
}

\section{A R T I C L E I N F O}

\section{Keywords:}

Postoperative pain

Morphine

Methadone

Opioids

Gynecological cancer

\begin{abstract}
A B S T R A C T
Study objective: The aim of this study was to compare methadone and morphine for the management of postoperative.

Design: Open, controlled study.

Setting: Postoperative recovering area, ward.

Patients: Sixty-four patients, ASA I-III, undergoing gynecological surgery for cancer.

Interventions: Morphine or methadone $0.15 \mathrm{mg} / \mathrm{kg}$ given preoperatively. After operation an intravenous morphine or intravenous methadone infusion at doses of $12 \mathrm{mg}$ /day was started.

Measurements: Pain intensity and opioid consumption.

Main results: Methadone infusion provided a better analgesia in comparison with morphine infusion on the second day. Opioid consumption was significantly lower in the methadone group. No episodes of relevant desaturation or signs of respiratory depression were recorded.

Conclusion: A preoperative bolus of methadone, followed by a continuous infusion of low doses post-operatively, provided a better analgesia, without adding risk of adverse effects, in comparison with morphine.
\end{abstract}

\section{Introduction}

Adequate postoperative pain control is an important part of perioperative medicine. Inappropriate perioperative pain management has numerous physiologic complications and is responsible of unnecessary suffering [1]. Regretfully, many patients remain undertreated. It has been reported that $>40 \%$ of patients report inadequate pain relief or pain of moderate intensity or greater in the postoperative period, despite treatment $[2,3]$. Of concern, unrelieved postoperative pain increases risk of developing chronic post surgical pain [1].

Intravenous patient-controlled analgesia (PCA) has become the mainstay for providing postoperative pain relief over the past years [4-6]. However, PCA may have some limitations. While PCA may reduce nursing time, the cost of pumps, disposables, and adverse events should be considered in balancing the overall cost-effectiveness of PCA.
[7]. For many years a long-available effective drug, such as methadone, has been underutilized. Despite methadone being a cost-effective analgesic for acute, chronic, neuropathic, and cancer pain, and can be administered via oral and parenteral routes [8], its use in the operating room and postoperatively is a neglected issue [7]. Only few old studies have assessed methadone for postoperative pain [9-12]. These studies were performed in a mixed population undergoing different surgeries and were principally based on a pre-operative administration of a methadone bolus.

The primary aim of this study trial was to compare the effects of methadone on pain scores with those of morphine for postoperative pain in patients undergoing a standard operation, like hysterosalpingectomy for gynecological cancer. In addition, the analgesic requirements and possible adverse effects induced by opioids were determined.

\footnotetext{
" This paper was written according to CONSORT recommendations (Fig. 1). The study was not registered, as it is not requested in our country. It was prepared before 2015, when most journals introduced the need to register trials. Moreover, there were no changes of the original protocol and all data of this trial have been included and were not replied in other studies, as redundant publication.

This research did not receive any specific grant from funding agencies in the public, commercial, or not-for-profit sectors.

* Corresponding author at: Anesthesia and Intensive Care Unit, Pain Relief and Palliative Care Unit, La Maddalena Cancer Center, Via san Lorenzo 312 , 90145 Palermo, Italy.

E-mail address: terapiadeldolore@lamaddalenanet.it (S. Mercadante).
} 


\section{Methods}

This was a randomized, active-controlled, study. The study protocol and statement of informed consent were approved by the institutional review board Ethical approval for this study (Ethical Committee $\mathrm{N}^{\circ} 6 /$ 2013) was provided by the Ethical Committee of the University of Palermo. All patients provided written informed consent. Patients were recruited in a period of two years.

Patients scheduled for open hystero-salpingectomy for gynecological cancer under general anesthesia were enrolled. Non-pregnant female patients were eligible for inclusion if they were at least 18 years old and had an American Society of Anesthesiologists physical status I to III. Patients were excluded if they were receiving opioids for chronic pain or for any other reason, had documented sleep apnea, alcohol or drug abuse, oxygen saturation of $<92 \%$, had any medical condition that would interfere with pain assessment. The use of any drug that would affect postoperative pain levels, such as gabapentinoids, steroids, or anti-inflammatory drugs was not allowed intraoperatively or postoperatively. Patients with a chronic pain condition necessitating treatment with these agents were also excluded from the study. Patients had to be withdrawn from the study if the oxygen saturation could not be maintained at $\geq 92 \%$ with supplemental oxygen $(3 \mathrm{~L} / \mathrm{min})$, if the respiratory rate could not be maintained at 8 breaths/min or greater, or if excessive sedation occurred.

\subsection{Procedures}

Patients scheduled for the first operation during the day (performed at $8 \mathrm{AM}$ ), were randomized by a computerized system to receive morphine (group 1) or methadone (group 2). A total of 64 participants were randomized chronologically in a 1:1 ratio according to the random coding table and were given the corresponding medication.

Patients were premedicated with midazolam $0.1 \mathrm{mg} / \mathrm{kg}$, plus morphine or methadone $0.15 \mathrm{mg} / \mathrm{kg}$, intravenously. During surgery, intravenous fentanyl was allowed as needed for analgesia. Nasogastric tube was not inserted. Following surgery, boluses of the study drugs in doses of $3 \mathrm{mg}$ were given to keep the patient comfortable in the postanesthesia care unit (PACU) if their pain intensity was $\geq 4 / 10$. These doses were computed in opioid consumption calculation on the operation day. Pain intensity was based on an 11-point numerical rating scale (NRS), where $0=$ no pain and $10=$ worst possible pain. Metoclopramide $5 \mathrm{mg}$ and clonidine $75 \mu \mathrm{g}$ were given in case of vomiting and shivering, respectively, during PACU stay.

At discharge from the PACU, an intravenous morphine or methadone infusion was given, at doses of $12 \mathrm{mg} /$ day. Paracetamol was given first at request of patients. Extra-doses of morphine or methadone in doses of $3 \mathrm{mg}$ were given as breakthrough medication. Patients were educated about asking for opioid analgesia when pain was considered no longer acceptable. The use of metoclopramide was permitted for controlling nausea and vomiting.

Comorbidities and some parameters such as, pain intensity (numerical scale 0-10), the level of nausea, drowsiness, and itching (from 0 to 3 ), episodes of desaturation $(<92 \%)$ were recorded since the patient was transferred to the surgical ward at $12 \mathrm{AM}$, and 2, 4, 6, 8, and $10 \mathrm{PM}$ on the operation day, labelled as T1, T2, T3, T4, T5, and T6, respectively. On the second day, these parameters were measured at 6 and $10 \mathrm{AM}$, and 2, 6, and $10 \mathrm{PM}$, labelled as $\mathrm{T} 7, \mathrm{~T} 8, \mathrm{~T} 9, \mathrm{~T} 10$, and $\mathrm{T} 11$, respectively. Opioid consumption was recorded in the PACU and in the surgical ward on the operation day, and on the second day.

The primary efficacy end point was the pain intensity at the different time intervals on operation day and on the second day. Secondary end points included the pain intensity differences (PID), and the time-weighted summed pain intensity difference over $48 \mathrm{~h}$ (SPID48), as well as the opioid consumption, as extra-doses of the study drugs given at patients' request.

\subsection{Statistical analysis}

To achieve the primary end point, a sample size of 20 patients per treatment group yields a statistical power of $80 \%$ with a Type 1 error of 0.05 , allowing the detection of a difference of 0.5 in mean pain intensity score (with a standard deviation of 0.5). The within-group standard deviation is assumed to be 3 , with a percentage of missing data of $20 \%$. The sample size was inflated by $40 \%$ to account for missing data, attrition, and protocol violations.

Statistical analysis of quantitative and qualitative data, including descriptive statistics, was performed for all items. Continuous data are expressed as mean \pm SD, unless otherwise specified. Frequency analysis was performed using the Pearson's chi-square test and Fisher exact test, as needed. The univariate analysis of variance (ANOVA) was performed to evaluate mean differences (age, weight, and opioid consumption) between patient groups. Whereas some variables were not normally distributed, we have used non-parametric tests and in particular the related-samples Friedman's two-way analysis of variance by ranks test (for intragroup comparison) and the independent-samples Mann-Whitney $U$ Test for intergroup comparison. Data were analyzed by IBM SPSS Software 22 version (IBM Corp., Armonk, NY, USA). All Pvalues were two-sided and $\mathrm{P}<0.05$ was considered statistically significant.

\section{Results}

Sixty-four patients met the inclusion criteria. Four patients had incomplete data. Thus, 60 patients were examined (32 and 28 patients in group 1 and 2, respectively). The flow diagram, according to CONSORT guidelines is reported in Fig. 1. The mean age was 56.3 years (SD 9.6), and the mean weight was $64.6 \mathrm{~kg}$ (SD 9.4). No differences between the two groups were found ( $P=0.799$ and 0.909 , respectively). Fifty, nine, and one patients were ASA II, III and I, respectively, with no differences between the groups (Fisher's exact test $P=0.209$ ). Also the distribution of comorbidities in morphine vs methadone group was similar for hypertension (12 vs 14 patients respectively, Fisher's exact test $\mathrm{P}=0.435$ ), diabetes ( 2 vs 5 patients respectively, Fisher's exact test $\mathrm{P}=0.235$ ), chronic respiratory disease (2 vs 1 patients respectively, Fisher's exact test $P=1.0$ ), and obesity ( 6 vs 3 patients respectively, Fisher's exact test $\mathrm{P}=0.482$ ). No episodes of relevant desaturation were recorded and no patient presented signs of respiratory depression.

The changes of parameters recorded at the different time intervals on the operation day and on the second day are shown in Tables 1 and 2. Pain intensity progressively changed in both groups. Methadone infusion provided a better analgesia in comparison with morphine infusion on the second day. PID at the various intervals and SPID48 are reported in Tables 3 and 4, respectively. The difference was significant for PID at the last measurements on the second day. Lower values of SPID48 were found in the methadone group, although the difference was not significant different between the two groups.

Intravenous paracetamol was used in 20 and 14 patients in morphine and methadone groups, respectively (Fisher's exact test $P=0.435$ ). Opioid consumption was significantly lower in the methadone group (Table 5).

\section{Discussion}

The findings of this study suggest that a preoperative dose of methadone, followed by a continuous infusion of low doses of methadone, provided better postoperative analgesia in patients undergoing in comparison with morphine in patients undergoing gynecological procedures. Moreover, analgesic consumption including paracetamol and study drugs was significantly lower in patients receiving methadone. This analgesic effect was not associated with more adverse effects among those commonly induced by opioids.

These findings are explained by several pharmacologic and clinical 


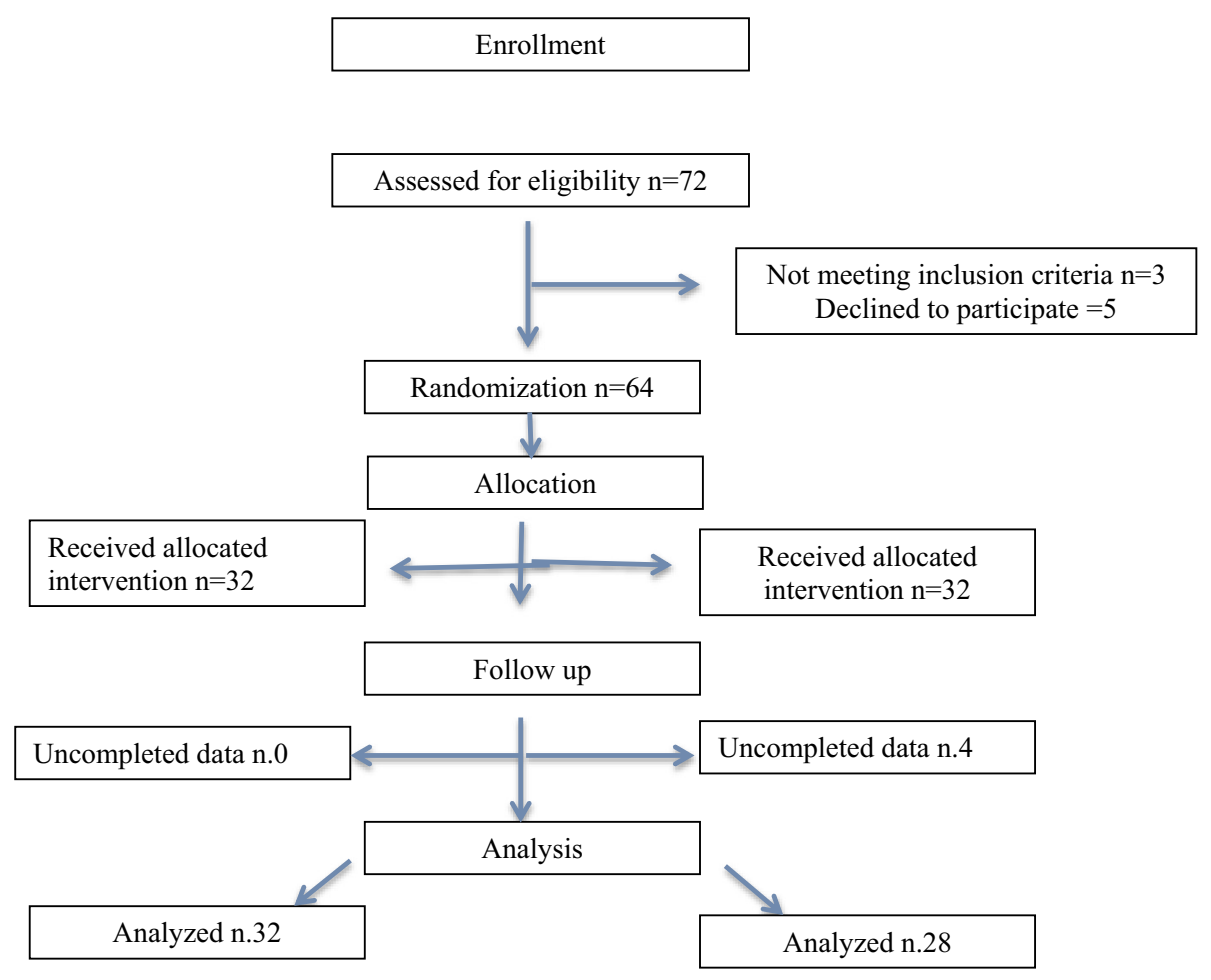

Fig. 1. CONSORT flow diagram.

Table 1

Changes of parameters recorded on the operative day (T1, T2, T3, T4, T5, T6 correspond to $12 \mathrm{AM}$, and 2, 4, 6, 8, and 10 PM, respectively.)

\begin{tabular}{|c|c|c|c|c|c|c|c|c|}
\hline & & $\mathrm{T} 1$ & $\mathrm{~T} 2$ & $\mathrm{~T} 3$ & $\mathrm{~T} 4$ & T5 & $\mathrm{T} 6$ & $\mathrm{P}$ intergroup \\
\hline \multirow[t]{2}{*}{ Pain intensity } & Morphine & $4.5(2.1)$ & $3.5^{\S}(2.4)$ & $2.3^{*}(1.9)$ & $1.6^{*}(2.1)$ & $0.9^{*}(1.4)$ & $0.6^{*}(1.2)$ & $\mathrm{T} 1 \mathrm{P}=0.018$ \\
\hline & Methadone & $3.0(2.2)$ & $2.1(2.3)$ & $1.2^{*}(1.4)$ & $0.8^{*}(1.3)$ & $0.5^{*}(1.2)$ & $0.3^{*}(0.9)$ & $\begin{array}{l}\mathrm{T} 2 \mathrm{P}=0.013 \\
\mathrm{~T} 3 \mathrm{P}=0.009\end{array}$ \\
\hline \multirow[t]{2}{*}{ Vomiting } & $\begin{array}{l}\text { Methadone } \\
\text { Morphine }\end{array}$ & $\begin{array}{l}0.04(0.2) \\
0.03(0.2)\end{array}$ & $\begin{array}{l}0.1(0.3) \\
0.06(0.2)\end{array}$ & $\begin{array}{l}0.1(0.3) \\
0.09(0.3)\end{array}$ & $\begin{array}{l}0.3(0.7) \\
0.0(0.0)\end{array}$ & $\begin{array}{l}0.1(0.4) \\
0.06(0.2)\end{array}$ & $\begin{array}{l}0.2(0.7) \\
0.06(0.2)\end{array}$ & NS \\
\hline & Methadone & $0.04(0.2)$ & $0.0(0.0)$ & $0.0(0.0)$ & $0.0(0.0)$ & $0.04(0.2)$ & $0.07(0.2)$ & NS \\
\hline Drowsiness & Morphine & $1.5(0.6)$ & $1.5(0.8)$ & $1.2(0.8)$ & $1.1(0.6)$ & $1.0^{\S}(0.7)$ & $0.5^{*}(0.5)$ & NS \\
\hline Itching & Methadone & $0.0(0.0)$ & $0.0(0.0)$ & $0.0(0.0)$ & $0.0(0.0)$ & $0.0(0.0)$ & $0.0(0.0)$ & NS \\
\hline
\end{tabular}

$\mathrm{P}<0.05$ vs T0.

P $<0.0005$ vs T0.

${ }^{5} \mathrm{P}<0.005$ vs T1, related-samples Friedman's two-way analysis of variance by ranks.

${ }^{\text {a }}$ Independent-samples Mann-Whitney $U$ test.

studies, although few trials assessed the value of methadone as a postoperative analgesic drug. It still remains a misunderstood drug [7], possibly due to some misconception about its use in the perioperative setting, due to its onset, duration, and metabolism. The onset of methadone is relatively fast, comparable to fentanyl, as central nervous system effect site methadone concentrations rapidly equilibrate with plasma concentrations [13], corresponding to the time course of miosis. Of interest, a lag time exists for the central nervous system compartment penetration and the onset time of morphine [14]. Thus methadone can be considered as a rapid-onset drug, with an onset time similar to that of fentanyl.

It has been reported that the duration of methadone analgesia is shorter than its elimination half-life. However, this observation is also true for other opioids as well other drugs $[15,16]$. Targeting doses and concentrations above the minimal analgesic concentration but below the threshold for respiratory depression, should provide a lasting analgesia and minimal central adverse effects. Of interest, a calibrated continuous infusion with low doses may overcome the problem, as it occurred in this study. Although methadone has a highly variable clearance and a large potential for drug interactions [17], these interferences are often subclinical $[7,18]$.

Pioneer investigations on methadone in the perioperative period were reported about 30 years ago [9-12]. An intravenous bolus of $20 \mathrm{mg}$ of methadone, after induction of anesthesia, determined a complete analgesia in approximately one-third of patients, and no analgesic requests during the 72 hour postoperative observation period were reported. Only one third of patients requested some postoperative opioid drugs, and the mean time to first opioid analgesic request was $20 \mathrm{~h}$. These outcomes were achieved without reporting relevant adverse effects.

After many years, data regarding the use of methadone in the perioperative setting still remain poor, particularly in abdominal cancer surgery. In patients undergoing hip arthroplasty a continuous infusion of $24 \mathrm{mg}$ /day of methadone with PCA boluses of $1 \mathrm{mg}$, determined a lower opioid consumption and less pain in comparison with morphine, while adverse effects were similar [19]. In a randomized trial, patients 
Table 2

Changes of parameters recorded on the first postoperative day (T7, T8, T9, T10, and T11 correspond to 6 and 10 AM, and 2, 6, and 10 PM, respectively).

\begin{tabular}{|c|c|c|c|c|c|c|c|}
\hline & & $\mathrm{T} 7$ & $\mathrm{~T} 8$ & T9 & $\mathrm{T} 10$ & $\mathrm{~T} 11$ & $\mathrm{P}$ intergroup ${ }^{\mathrm{a}}$ \\
\hline \multirow[t]{5}{*}{ Pain intensity } & Morphine & $2.3(1.8)$ & $2.0(1.6)$ & $1.0^{\S}(1.1)$ & $1.1^{\S}(1.4)$ & $0.5^{*}(0.8)$ & $\mathrm{T} 7 \mathrm{P}<0.0005$ \\
\hline & Methadone & $0.6(1.0)$ & $0.6(1.6)$ & $0.3(0.7)$ & $0.07(0.4)$ & $0.0(0.0)$ & T8 $\mathrm{P}<0.0005$ \\
\hline & & & & & & & T9 $\mathrm{P}=0.003$ \\
\hline & & & & & & & $\mathrm{T} 10 \mathrm{P}<0.0005$ \\
\hline & & & & & & & $\mathrm{T} 11 \mathrm{P}=0.003$ \\
\hline \multirow[t]{2}{*}{ Nausea } & Morphine & $0.4(0.7)$ & $0.2(0.4)$ & $0.03(0.2)$ & $0.09(0.4)$ & $0.03(0.2)$ & NS \\
\hline & Methadone & $0.5(0.9)$ & $0.3(0.5)$ & $0.1(0.6)$ & $0.07(0.3)$ & $0.1(0.4)$ & \\
\hline \multirow[t]{2}{*}{ Vomiting } & Morphine & $0.2(0.4)$ & $0.0(0.0)$ & $0.03(0.2)$ & $0.03(0.2)$ & $0.0(0.0)$ & NS \\
\hline & Methadone & $0.1(0.4)$ & $0.1(0.3)$ & $0.07(0.3)$ & $0.04(0.2)$ & $0.04(0.2)$ & NS \\
\hline \multirow[t]{2}{*}{ Drowsiness } & Morphine & $0.7(0.5)$ & $0.7(0.7)$ & $0.4(0.6)$ & $0.2^{\#}(0.5)$ & $0.1^{\#}(0.4)$ & NS \\
\hline & Methadone & $0.6(0.6)$ & $0.4(0.5)$ & $0.2(0.4)$ & $0.2(0.4)$ & $0.1(0.3)$ & NS \\
\hline \multirow[t]{2}{*}{ Itching } & Morphine & $0.0(0.0)$ & $0.0(0.0)$ & $0.0(0.0)$ & $0.0(0.0)$ & $0.0(0.0)$ & NS \\
\hline & Methadone & $0.0(0.0)$ & $0.04(0.2)$ & $0.04(0.2)$ & $0.0(0.0)$ & $0.0(0.0)$ & NS \\
\hline
\end{tabular}

* $\mathrm{P}<0.001$ vs T0.

${ }^{*} \mathrm{P}<0.0005$ vs T0.

${ }^{\S} \mathrm{P}<0.005$ vs T0.

P $<0.05$ vs T0; related-samples Friedman's two-way analysis of variance by ranks.

a Independent-samples Mann-Whitney $U$ test.

Table 3

Pain intensity difference at the selected intervals.

\begin{tabular}{|c|c|c|c|c|c|c|c|c|c|c|c|c|}
\hline & & \multicolumn{6}{|c|}{ Operation day } & \multicolumn{5}{|c|}{ Second day } \\
\hline & & $\mathrm{T} 1-\mathrm{T} 2$ & T2-T3 & T3-T4 & T4-T5 & T5-T6 & T1-T6 & T7-T8 & T8-T9 & T9-T10 & T10-T11 & T7-T11 \\
\hline Morphine & Mean (SD) & $1.0(2.4)$ & $1.2(2.1)$ & $0.7(1.7)$ & $0.72(1.6)$ & $0.3(1.1)$ & $3.84(2.1)$ & $0.3(2.0)$ & $0.9(1.4)$ & $0.0(1.6)$ & $0.6(1.0)$ & $1.8(1.7)$ \\
\hline Methadone & Mean (SD) & $0.9(2.5)$ & $0.9(1.9)$ & $0.4(0.9)$ & $0.3(1.2)$ & $0.2(0.5)$ & $2.7(2.4)$ & $0.1(1.8)$ & $0.3(1.5)$ & $0.3(0.8)$ & $0.1(0.4)$ & $0.6(1.0)$ \\
\hline $\mathrm{P}$ intergroup ${ }^{\mathrm{a}}$ & & 0.826 & 0.487 & 0.152 & 0.210 & 0.758 & 0.057 & 0.767 & 0.007 & 0.835 & 0.006 & 0.005 \\
\hline
\end{tabular}

${ }^{\text {a }}$ Independent-samples Mann-Whitney $U$ test.

Table 4

SPID 48 in morphine and methadone groups.

\begin{tabular}{|c|c|c|c|c|c|c|c|c|}
\hline & \multirow[t]{2}{*}{$\mathrm{N}$} & \multirow[t]{2}{*}{ Mean } & \multirow[t]{2}{*}{ Std. deviation } & \multirow[t]{2}{*}{ Std. error } & \multicolumn{2}{|c|}{$95 \%$ confidence interval for mean } & \multirow[t]{2}{*}{ Minimum } & \multirow[t]{2}{*}{ Maximum } \\
\hline & & & & & Lower bound & Upper bound & & \\
\hline Morphine & 32 & 29.2188 & 20.82451 & 3.68129 & 21.7107 & 36.7268 & -20.00 & 62.00 \\
\hline Methadone & 28 & 23.5714 & 21.58262 & 4.07873 & 15.2026 & 31.9403 & -23.00 & 64.00 \\
\hline Total & 60 & 26.5833 & 21.19185 & 2.73586 & 21.1089 & 32.0578 & -23.00 & 64.00 \\
\hline
\end{tabular}

$\mathrm{P}=0.378$, independent-samples Mann-Whitney $U$ test.

Table 5

Opioid consumption in the two groups; univariate analysis of variance (ANOVA test).

\begin{tabular}{llllll}
\hline & & N & Mean & DS & P \\
\hline \multirow{2}{*}{ Operation day } & Morphine mg & 32 & 6,09 & 4,067 & 0.002 \\
& Methadone mg & 28 & 3,11 & 2,998 & \\
\multirow{2}{*}{ Second day } & Morphine mg & 32 & 2,25 & 2,851 & $<0.0005$ \\
& Methadone mg & 28 & 0,11 & 0,567 & \\
\hline
\end{tabular}

undergoing vertebral surgery, received an initial loading dose of $0.75 \mu \mathrm{g} / \mathrm{kg}$ sufentanil before surgical incision and a sufentanil infusion of $0.25 \mu \mathrm{g} / \mathrm{kg} / \mathrm{h}$ or methadone infusion, $0.2 \mathrm{mg} / \mathrm{kg}$, after intubation. In comparison with sufentanil, methadone significantly reduced the opioid consumption and pain scores in the postoperative period [20]. In a similar surgical population, preoperative methadone $0.2 \mathrm{mg} / \mathrm{kg}$ conferred a longer analgesia and less opioid consumption in comparison with hydromorphone $2 \mathrm{mg}$, given at the end of surgery [21,22], although the doses chosen (about $15 \mathrm{mg}$ and $2 \mathrm{mg}$, for methadone and hydromorphone, respectively) should not be considered to be equivalents [23]. According to these data, an initial priming with methadone provided a prolonged and effective analgesia. These effects were attributed to the slow rate of methadone elimination, and a $N$-methyl-D- aspartate receptor antagonism [7].

In a gynecological cancer population similar to that examined in this study, methadone and morphine in doses of $20 \mathrm{mg}$ were given after induction of anesthesia. Patients who were administered methadone required less methadone than morphine in the recovery room and on the wards, and reported lower pain intensity scores [24]. Similarly, in women undergoing abdominal hysterectomy, methadone or morphine, $0.25 \mathrm{mg} / \mathrm{kg}$ were given preoperatively with further increments in the recovery room for analgesia. Patients in the methadone group had lower pain scores in the subsequent $48 \mathrm{~h}$ and had a less opioid consumption [25]. Taken together, these data are consistent with a sustained therapeutic analgesic effect in the postoperative period. In the present study, a preoperative bolus of methadone and a postoperative infusion of low doses provided superior analgesia over morphine, suggesting that methadone is an effective and safe drug for postoperative pain. Of interest, intravenous infusion of methadone has been used for cancer pain management in opioid-tolerant patients, successfully [8].

There are some limitations of this study. Only women and a typical gynecologic surgery were chosen. Gynecological procedures were chosen as they are standard operations in terms of duration and potential capacity to produce postoperative pain.

In conclusion, a preoperative bolus of methadone, followed postoperatively by a continuous infusion of low doses provided a potent 
analgesia, without adding risk of adverse effects, in comparison with morphine. More information is needed about the effectiveness and safety in outpatients, those who are opioid tolerant, and the cost-effectiveness of methadone in comparison with other opioids or conventional PCA in the different surgeries. Nevertheless, the findings of this study suggest that it is opportune to reappraise the use of methadone in the perioperative period.

\section{Declaration of competing interest}

None.

\section{References}

[1] Kehlet H, Jensen TS, Woolf CJ. Persistent postsurgical pain: risk factors and prevention. Lancet 2006;367:1618-25.

[2] Brennan F, Carr DB, Cousins M. Pain management: a fundamental human right. Anesth Analg 2007;105:205-21.

[3] Moss E, Taverner T, Norton P, Lesser P, Cole P. A survey of postoperative pain management in fourteen hospitals in the UK. Acute Pain 2005;7:13-20.

[4] Rathmell JP, Wu CL, Sinatra RS, Ballantyne JC, Ginsberg B, Gordon DB, et al. Acute post-surgical pain management: a critical appraisal of current practice. Reg Anesth Pain Med 2006;31(Suppl. 1):1-42.

[5] Taylor A, Stanbury L. A review of postoperative pain management and the challenges. Curr Anaesth Crit Care 2009;20:188-94.

[6] Hudcova J, McNicol E, Quah C, Lau J, Carr DB. Patient controlled opioid analgesia versus conventional opioid analgesia for postoperative pain. Cochrane Database Syst Rev 2006:CD003348.

[7] Kharasch ED. Intraoperative methadone: rediscovery, reappraisal, and reinvigoration? Anesth Analg 2011;112:13-6. https://doi.org/10.1213/ANE. 0b013e3181fec9a3.

[8] Mercadante S, Knotkova H. Intravenous use of methadone: efficacy and safety. In: Cruciani RA, editor. Handbook of methadone prescribing and buprenorphine therapy. Berlin: Springer; 2013. p. 81-9.

[9] Gourlay GK, Willis RJ, Wilson PR. Postoperative pain control with methadone: influence of supplementary methadone doses and blood concentration-response relationships. Anesthesiology 1984;61:19-26.

[10] Gourlay GK, Plummer JL, Cherry DA, Cousins MJ. Does intravenous methadone provide longer lasting analgesia than intravenous morphine? Pain 1990;42:383-6.

[11] Gourlay GK, Willis RJ, Lamberty J. A double-blind comparison of the efficacy of methadone and morphine in postoperative pain control. Anesthesiology 1986;64:322-7.
[12] Gourlay GK, Willis RJ, Wilson PR. Postoperative pain control with methadone: influence of supplementary methadone doses and blood concentration-response relationships. Anesthesiology 1984;61:19-26.

[13] Inturrisi CE, Colburn WA, Kaiko RF, Houde RW, Foley KM. Pharmacokinetics and pharmacodynamics of methadone in patients with chronic pain. Clin Pharmacol Ther 1987;41:392-401.

[14] Gourlay GK, Wilson PR, Glynn CJ. Pharmacodynamics and pharmacokinetics of methadone during the perioperative period. Anesthesiology 1982;57:458-67.

[15] Grochow L, Sheidler V, Grossman S, Green L, Enterline J. Does intravenous methadone provide longer lasting analgesia than intravenous morphine? A randomized, double-blind study. Pain 1989;38:151-7.

[16] Dahan A, Aarts L, Smith TW. Incidence, reversal, and prevention of opioid-induced respiratory depression. Anesthesiology 2010;112:226-38. https://doi.org/10. 1097/ALN.0b013e3181c38c25.

[17] Mercadante S. Opioid metabolism and clinical aspects. Eur J Pharmacol 2015;769:71-8. https://doi.org/10.1016/j.ejphar.2015.10.049.

[18] Kharasch ED, Hoffer C, Whittington D, Walker A, Bedynek PS. Methadone pharmacokinetics are independent of cytochrome P4503A (CYP3A) activity and gastrointestinal drug transport. Insights from methadone interactions with ritonavir/ indinavir. Anesthesiology 2009;110:660-72. https://doi.org/10.1097/ALN. 0b013e3181986a9a.

[19] Neto JO, Machado MD, de Almeida Correa M, Scomparim HA, Posso IP, Ashmawi HA. Methadone patient-controlled analgesia for postoperative pain: a randomized, controlled, double-blind study. J Anesth 2014;28:505-10. https://doi.org/10. 1007/s00540-013-1785-3.

[20] Gottschalk A, Durieux ME, Nemergut EC. Intraoperative methadone improves postoperative pain control in patients undergoing complex spine surgery. Anesth Analg 2011;112:218-23. https://doi.org/10.1213/ANE.0b013e3181d8a095.

[21] Murphy GS, Szokol JW, Avram MJ, Greenberg SB, Marymont JH, Shear T, et al Intraoperative methadone for the prevention of postoperative pain: a randomized, double-blinded clinical trial in cardiac surgical patients. Anesthesiology 2015;122:1112-22. https://doi.org/10.1097/ALN.0000000000000633.

[22] Murphy GS, Szokol JW, Avram MJ, Greenberg SB, Shear TD, Deshur MA, et al. Clinical effectiveness and safety of intraoperative methadone in patients undergoing posterior spinal fusion surgery: a randomized, double-blinded, controlled trial. Anesthesiology 2017;126:822-33. https://doi.org/10.1097/ALN 0000000000001609.

[23] Mercadante S, Caraceni A. Conversion ratios for opioid switching in the treatment of cancer pain: a systematic review. Palliat Med 2011;25. https://doi.org/10.1177/ 0269216311406577. 504-1.

[24] Richlin DM, Reuben SS. Postoperative pain control with methadone following lower abdominal surgery. J Clin Anesth 1991;3:112-6.

[25] Chui PT, Gin T. A double-blind randomised trial comparing postoperative analgesia after perioperative loading doses of methadone or morphine. Anaesth Intensive Care 1992;20. 46-5. 